\section{Steroide in der COPD-Therapie}

\section{Einleitung}

Die COPD ist charakterisiert a) durch eine langsam über Jahre und Jahrzehnte progredient zunehmende Atemwegsobstruktion, die in ihrem Ausprägungsgrad nur wenig variabel ist, und b) durch fakultativ vorliegende Sekundärveränderungen, wie in erster Linie dem Lungenemphysem aber auch einer Rechtsherzbelastung und in schweren Fällen einer respiratorischen Insuffizienz [1]. Pathophysiologisch liegt der COPD eine meist durch inhalative Noxen (insbesondere Zigarettenrauch, aber auch berufliche Staubexposition) ausgelöste bronchiale Entzündungsreaktion der zentralen und peripheren Atemwege mit Beteiligung des Lungenparenchyms zugrunde [2].

Basierend auf der bei der COPD beschriebenen persistierenden bronchialen Entzündungsreaktion und vor dem Hintergrund des meist guten Ansprechens beim Asthma bronchiale lag der Gedanke nahe, bei den betroffenen Patienten eine weitere Verschlechterung der Lungenfunktion durch die Gabe von inhalativen Glukokortikosteroiden $\mathrm{zu}$ unterbinden und dadurch die Exazerbations- und die Mortalitätsrate bei gleichzeitiger Verbesserung der Lebensqualität zu senken, was sich bisher in den entsprechenden nationalen und internationalen Empfehlungen zur Therapie der COPD niederschlug [1,3-7]. Nach Jahrzehnten der intensiven Forschung im Bereich des Asthma bronchiale wird erst in jüngster Zeit zunehmend akzeptiert, dass sich die positiven Erfahrungen mit der Steroidlangzeittherapie beim Asthma bronchiale keineswegs auf die COPD übertragen lassen [8-11].

Bezüglich der Eignung von Glukokortikosteroiden zur Therapie der COPD muss zwischen den Effekten bei klinisch stabilen Patienten und in der Notfallsituation (Exazerbation) unterschieden werden.

\section{Glukokortikosteroide bei der stabilen COPD}

Auf die Fragen, ob bei der stabilen COPD, die gekennzeichnet ist a) durch eine Reversibilität der Atemwegsobstruktion $\left(\mathrm{FEV}_{1}\right)$ von $<15 \%$ nach Inhalation eines kurzwirksamen $\beta_{2}$ Agonisten, und b) durch eine mehrmonatige exazerbationsfreie Erkrankungsphase, eine Dauertherapie mit inhalativen Glukokortikosteroiden indiziert ist, wurde in der Vergangen-

Pneumologie 2000; 54: 474-477

(c) Georg Thieme Verlag Stuttgart · New York ISSN 0934-8387

\section{A. Gillissen \\ Medizinische Klinik und Poliklinik II, Universitätsklinik der Rheinischen Friedrich-Wilhelms-Universität Bonn}

heit mehrfach Stellung bezogen [12-18]. In Langzeittherapiestudien $(1 / 2-3$ Jahre) mit inhalativen Glukokortikosteroiden zeigte sich bei Subgruppen a) ein, bezogen auf die gesamte Studienzeit allenfalls schwach-signifikanter Effekt im Sinne einer Reduktion des $\mathrm{FEV}_{1}$-Abfalls über einen Studienraum von maximal 3 Jahren, der aber nur aus dem entsprechenden Effekt der ersten Behandlungsmonate (bis zu einem $1 / 2$ Jahr) resultierte, b) einen, falls untersucht, guten Effekt auf die Verbesserung physischer Leistungsparameter (nur bezogen auf die ersten sechs Monate), und c) bei älteren Arbeiten zwar eine teilweise gute Verbesserung der Lungenfunktion, wobei aber die Studienergebnisse wegen eines nicht stringenten Ausschlusses von Patienten mit einem Asthma bronchiale als zweifelhaft gewertet werden müssen [19-33].

Die Non-Responderrate der mit inhalativen Glukokortikosteroiden behandelten COPD-Patienten ist mit 80-90\% ausgesprochen hoch, so dass diese Medikamentengruppe als grundsätzlich ungeeignet für die breite Anwendung bei stabilen COPD-Patienten angesehen werden muss [31,34]. Eine entsprechende Angleichung der meist älteren Therapieempfehlungen wird daher in der nächsten Zeit erfolgen und wurde kürzlich durch die auf den Konferenzen der European Respiratory Society (ERS) 1999 in Madrid und der American Respiratory Society (ATS) 2000 in Toronto vorgestellten GOLD-Initiative (Global Initiative for chronic obstructive Lung Disease) in Form eines noch nicht in schriftlicher Form publizierten internationalen Konsensuspapieres initiiert $[3,4,35,36]$.

Es gibt Hinweise, dass insbesondere Patienten mit einer „Asthma-ähnlichen“ Erkrankungsform einen besonderen Nutzen von der Therapie mit Glukokortikosteroiden zu haben scheinen [37]. Da in der pneumologischen Praxis oft COPD/ Asthma bronchiale Mischformen vorkommen, unklar ist, welche COPD-Schweregrade einen besonderen Nutzen von einer Steroidtherapie haben, es keine Dosis-Nutzen-Relationsstudien gibt, und Responder von Non-Respondern vor Therapiebeginn nicht voneinander zu unterscheiden sind, kann der klinische Nutzen nur durch die testweise Applikation von inhalativen Glukokortikosteroiden über einen definierten Zeitraum mit regelmäßigen Reevaluationen (z.B. quartalsweise) erfolgen. Gegebenenfalls ist auch ein Absetzen einer initialen Steroidtherapie sinnvoll, was einen späteren erneuten Therapieversuch nicht unbedingt ausschließen muss [38]. Ein entsprechender Vorschlag zum Einsatz von inhalativen Glukokortikosteroiden bei Patienten mit einer stabilen COPD und der Überprüfung der Therapieeffektivität wurde kürzlich in dieser Zeitschrift publiziert. Bezüglich der Details wird 
daher auf den dort formulierten Vorschlag, der nach gegenwärtigem Kenntnisstand auch in modifizierter Form in den GOLD-Empfehlungen Eingang finden wird, verwiesen [15].

\section{Glukokortikosteroide bei der akuten Exazerbation der COPD}

Der Einsatz von systemischen Glukokortikosteroiden in der Behandlung der akuten Exazerbation einer COPD ist dagegen unstrittiger. Emerman et al. fanden zwar bei 96 COPDPatienten, dass eine systemische Glukokortikosteroidapplikation $5 \mathrm{~h}$ nach Therapiebeginn die Häufigkeit einer dann notwendigen stationären Aufnahme nicht reduzieren konnte [39]. Auf der anderen Seite führte bei 113 COPD-Patienten mit einer akuten Exazerbation eine frühzeitige Steroidgabe $\mathrm{zu}$ einer zumindest schwach signifikanten $(p<0,05)$ Lungenfunktionsverbesserung (Plazebo): Peak-Flow-Anstieg um 21,7 l/ min (5,5 l/min); $\mathrm{FEV}_{1}$-Anstieg um 0,14l (0,02 l) [40]. Bezogen auf die Lungenfunktion scheint sich der positive Effekt einer oralen oder intravenösen Glukokortikosteroidtherapie überwiegend auf die ersten, z.B. drei Behandlungstage nach der stationären Aufnahme auszuwirken [41]. Thompson et al. behandelten 27 akut exazerbierte COPD-Patienten mit initial oral $60 \mathrm{mg}$ Prednisolon, gefolgt von einer Reduktion in 20mg-Schritten. Am 10. Behandlungstag wurde die Steroidtherapie ganz abgesetzt [42]. Gegenüber Plazebo verbesserte sich die $\mathrm{FEV}_{1}$ und der $\mathrm{PaO}_{2}$ am Tag 3 nach Therapiebeginn schwach $(\mathrm{p}<0,05)$, am 10. Tag deutlich signifikant $(\mathrm{p}<0,01)$. Die mittels Fragebogen quantifizierte Dyspnoeskala fiel bei der VerumGruppe an den Tagen 2-5 signifikant gegenüber der Plazebo besser aus. Prednisolon führte auch $\mathrm{zu}$ einer geringeren Senkung der Therapieversagerrate und der notwendigen kurzfristigen Wiederaufnahmen in der Notfallaufnahme $(p<0,002)$. Die in der „Veterans Affairs Cooperative Study Group“-Studie an 271 COPD-Patienten mit einer schweren COPD $\left(\mathrm{FEV}_{1}<1 \mathrm{l}\right)$ erhobenen Ergebnisse bestätigen diesen positiven Trend [43]. Hier erhielten die Patienten über einen Zeitraum von $72 \mathrm{~h}$ eine hohe Dosis von je $125 \mathrm{mg}$ Methylprednisolon i.v. alle $6 \mathrm{~h}$, mit anschließender schrittweiser Reduktion und Absetzen der oral weitergeführten Steroidtherapie am 15. bzw. 57. Behandlungstag. Gegenüber der Plazebogruppe imponierte die Steroidgruppe durch eine verkürzte mittlere Krankenhaus-Behandlungszeit (8,5 vs. 9,7 Tage, $\mathrm{p}=0,03$ ), durch eine geringe Zahl an Therapieversagern (am Tag 30: 23 vs. 33\%, am Tag 90: 37 vs. $48 \%$ ) und durch einen allerdings nur an den Tagen $1-3$ nach Behandlungsbeginn signifikant $(p<0,05)$ schnelleren $F V_{1}$-Anstieg. Die beiden Steroidgruppen unterschieden sich dabei in ihrer Effektivität nicht. An Nebenwirkungen wurde von den Autoren der steroidbedingte Anstieg der Hyperglykämierate hervorgehoben [43]. In einer kleineren ebenfalls kürzlich publizierten Studie erhielten 56 exazerbierte COPD-Patienten während eines Zeitraums von 14 Tagen neben der Standardtherapie, bestehend aus Bronchodilatatoren und Antibiotika, zusätzlich oral Prednisolon (30 mg) oder Plazebo [44]. Die FEV 1 verbesserte sich von 28,2 auf 41,5\% (Plazebo: 25,7 auf 32,2\%) bzw. in einem 5-Tageszeitraum nach stationärer Aufnahme um $90 \mathrm{ml} /$ Tag (Plazebo: $30 \mathrm{ml} / \mathrm{Tag}$ ). Im Gegensatz dazu unterschieden sich die Plazebo- und Steroidgruppen 6 Wochen nach Therapie nicht mehr.

Zusammenfassend ist die Therapie der akuten Exazerbation mit systemischen Glukokortikosteroiden sinnvoll und durch entsprechende Studienergebnisse untermauert. $\mathrm{Zu}$ berücksichtigen ist, dass der überwiegende Benefit in den ersten Tagen zu erwarten ist, insbesondere systemisch (oral, intervenös) applizierte Glukokortikosteroide mit einem erheblichen Nebenwirkungspotential behaftet sind und hohe gegenüber niedrigen Initialdosen keinen wesentlichen Vorteil zu besitzen scheinen. Somit entspricht die Pharmakotherapie der akuten Exazerbation der COPD in etwa der des Status asthmaticus $[4,45]$. Zwar sind sich die nationalen und internationalen Empfehlungen dahingehend beim Management der akuten Exazerbation der COPD einig, dass systemische Glukokortikosteroide zur Notfallmedikation gehören, unklar ist aber, welche Applikationsform empfohlen werden soll, obwohl die intravenöse gegenüber der oralen Gabe der Vorzug gegeben wird, und welche Dosis sinnvoll erscheint. Weitgehende Einigkeit besteht in der Therapiedauer von wenigen Wochen, um bei fraglichem Nutzen bei einer längeren Anwendung das Auftreten typischer Nebenwirkungen zu vermeiden $[3,4,6,36]$. Sinnvoll erscheint daher bei einer akuten Exazerbation die initiale systemische Glukokortikosteroid-Gabe von $30 \mathrm{mg} /$ Tag bis max. $100 \mathrm{mg} /$ Tag. Die Therapiedauer sollte einen Zeitraum von 1 - 2 Wochen nicht überschreiten.

\section{Resümee}

Inhalative Glukokortikosteroide sind zur Therapie der stabilen COPD bei den meisten (80-90\%) aller Patienten ungeeignet. Nur mittels regelmäßiger Therapiekontrollen, die die Basis der Entscheidung über die Fortsetzung, das Absetzen oder über eine Therapiepause bilden, kann im individuellen Fall über den Sinn einer solchen Therapiemaßnahme entschieden werden. Im Fall einer akuten Exazerbation ist die systemische Applikation von Glukokortikosteroiden sinnvoll, sofern die Therapiedauer von im Regelfall 1-2 Wochen nicht überschritten wird. Die inhalativ oder systemisch durchgeführte Glukokortikosteroidtherapie muss, angepasst an die individuelle Erkrankungssituation, eingebettet sein in eine ergänzende Pharmakotherapie, wie z.B. mit Bronchodilatatoren $\left(\beta_{2^{-}}\right.$ Agonisten, Parasympatholytika, Theophyllin), Antibiotika, und weitere unterstützende und präventive Maßnahmen, wie z.B. Förderung der mukoziliären Clearance, $\mathrm{O}_{2}$-Gaben, invasive/ nicht-invasive Beatmung, Impfungen, Schulungsmaßnahmen inkl. Zigarettenentwöhnungsprogramme und Rehabilitation.

\section{Literatur}

${ }^{1}$ Siafakas NM, Vermeire P, Pride NB, Paoletti P, Gibson J, Howard P, Yernault JC, Decramer M, Higenbottam T, Postma DS, Rees J. Optimal assessment and management of chronic obstructive pulmonary disease (COPD). Eur Respir J 1995; 8: 1398-1420

${ }^{2}$ Gillissen A. Pathogenese der COPD. In: Gillissen A (Hrsg.). Die chronisch obstruktive Lungenerkrankung. Bremen: Uni-Med Verlag, 2000

${ }^{3}$ American Thoracic Society. Standards for the diagnosis and care of patients with chronic obstructive pulmonary disease. Am J Respir Crit Care Med 1995; 152: S77-S120

${ }^{4}$ British Thoracic Society. Guidelines for the management of chronic obstructive pulmonary disease. Thorax 1997; 52: S1 S28

${ }^{5}$ Deutsche Atemwegsliga. Vorschläge zur Diagnostik und Therapie des chronischen Cor Pulmonale. Pneumologie 1994; 48: $287-$ 291 
${ }^{6}$ Fabbri LM, Caramori G, Beghe B, Papi A, Ciaccia A. Chronic obstructive pulmonary disease international guidelines. Curr Opin Pulm Med 1998; 4: 76 -84

${ }^{7}$ Leuenberger P, Anderhub HP, Brändli O, Keller R, Knoblauch A, Kuhn M, Perruchoud AP, Rochat T, Russi E, Villiger B, Zellweger JP. Management 1997 of chronic obstructive pulmonary disease. Schweiz Med Wochenschr 1997; 127: 766- 782

8 Schayck van CP, Grunsven van PM, Dekhuijzen PNP. Do patients with COPD benefit from treatment with inhaled corticosteroids. Eur Respir J 1996; 9: 1969-1972

${ }^{9}$ Calverley PMA. Re-assessing the evidence about inhaled corticosteroids in chronic obstructive pulmonary disease. Thorax 1999; 54: $3-4$

${ }^{10}$ Barnes PJ. New therapies for chronic obstructive pulmonary disease. Thorax 1998; 53: 137-147

${ }^{11}$ Wedzicha JA. Inhaled corticosteroids in COPD: awaiting controlled trials. Thorax 1993; 48: 305-307

${ }^{12}$ Gillissen A. Kortisontherapie bei chronisch-obstruktiver Bronchitis - Contra-Position. Dtsch Med Wschr 2000; 125: 222 - 223

${ }^{13}$ Vogelmeier C. Kortisontherapie bei chronisch-obstruktiver Bronchitis - Pro-Position. Dtsch Med Wschr 2000; 125: 220-221

${ }^{14}$ Schäfer H, Ewig S, Gillissen A. Therapeutische Optionen im Management der stabilen chronisch obstruktiven Lungenerkrankung (COPD). Dtsch Med Wschr 2000; 125: 230-235

${ }^{15}$ Gillissen A, Buhl R, Kardos P, Magnussen H, Matthys H, Rabe KF, Rothe T, Russi EW, Schuaer J, Schmitz M, Vogelmeier C, Wettengel R, Worth H, Menz G. Inhalierbare Kortikosteroide in der Langzeittherapie der COPD. Stellungnahme eines Expertengremiums. Pneumologie 2000; 54: 256 - 262

16 Burge PS. EUROSCOP, ISOLDE and the Copenhagen City Lung Study. Thorax 1999; 54: 287-288

${ }^{17}$ Calverley PMA. Inhaled Corticosteroids are beneficial in chronic obstructive pulmonary disease. Am J Respir Crit Care Med 2000; 161: $341-344$

${ }^{18}$ Ewig S, Schäfer $\mathrm{H}$. Medikamentöse Therapie der COPD. In: Gillissen A (Hrsg.). Die chronisch obstruktive Lungenerkrankung. Bremen: Uni-Med Verlag, 2000: 84-95

${ }^{19}$ Bourbeau J, Rouleau MY, Boucher S. Randomised controlled trial of inhaled corticosteroids in patients with chronic obstructive pulmonary disease. Thorax 1998; 53: 477-482

${ }^{20}$ Chanez P, Vignola AM, O'Shaugnessy T, Enander I, Li D, Jeffery PK, Bousquet J. Corticosteroid reversibility in COPD is related to features of asthma. Am J Respir Crit Care Med 1997; 155: 15291534

${ }^{21}$ Cox G, Whitehead L, Dolovich M, Jordana M, Gauldie J, Newhouse MT. A randomized controlled trial on the effect of inhaled corticosteroids on airways inflammation in adult cigarette smokers. Chest 1999; 115: $1271-1277$

${ }^{22}$ Dompeling E, Schayck van CP, Grunsven van PM, Herwaarden van CLA, Akkermans RP, Molema J, Folgering H, Weel van C. Slowing the deterioration of asthma and chronic obstructive pulmonary disease observed during bronchodilator therapy by adding inhaled corticosteroids. Ann Intern Med 1993; 118: 770 778

${ }^{23}$ Grunsven van PM, Schayck van CP, Derenne JP, Kerstjens HAM, Renkema TEJ, Postma DS, Similowski T, Akkermans RP, Pasker-de Jong PCM, Dekhuijzen PNP, Herwaarden van CLA, Weel van C. Long term effects of inhaled corticosteroids in chronic obstructive pulmonary disease: a meta-analysis. Thorax 1999; 54: 7-14

${ }^{24}$ Lepage T, Bourcereau J, Terrioux P, Velicitat P, Roche N. Influence des résultats du test de réversibilité de l'obstruction bronchique sur la prise en charge de bronchopneumopathies chroniques obstructives (BPCO) en practique pneumologique. Rev Mal Respir 2000; 17: S25

${ }^{25}$ Nishimura K, Koyama H, Ikeda A, Tsukino M, Hajiro T, Mishima $\mathrm{M}$, Izumi T. The effect of high-dose inhaled beclomethasone dipropionate in patients with stable COPD. Chest 1999; 115: 31 37

${ }^{26}$ Pauwels RA, Lofdahl C-G, Laitinen LA, Schouten JP, Postma DS, Pride NB, Ohlsson SV. Long-term treatment with inhaled budesonide in persons with mild chronic obstructive pulmonary disease who continue smoking. N Engl J Med 1999; 340: $1948-$ 1953

${ }^{27}$ Kerstjens HAM, Brand PLP, Hughes MD, Robinson NJ, Postma DS, Sluiter HJ, Bleecker ER, Dekhuijzen PNP, de Jongh PM, Mengelers HJJ, Overbeek SE, Schoonbrood DFME. A comparison of bronchodilator therapy with or without inhaled corticosteroid therapy for obstructive airways disease. N Engl J Med 1992; 327: $1413-1419$

28 Pizzichini E, Pizzichini MMM, Gibson P, Parameswaran K, Gleich GJ, Berman P, Dolovich J, Hargreave FE. Sputum eosinophilia predicts benefit from prednisone in smokers with chronic obstructive bronchitis. Am J Respir Crit Care Med 1998; 158: $1511-1517$

${ }^{29}$ Renkema TEJ, Schouten JP, Koeter GH, Postma DS. Effects of long-term treatment with corticosteroids in COPD. Chest 1996; 109: $1156-1162$

${ }^{30}$ Shim CS, Williams Jr MH. Aerosol beclomethasone in patients with steroid-responsive chronic obstructive pulmonary disease. Am J Med 1985; 78: 655-658

${ }^{31}$ Vestbo J, Sørensen T, Langer P, Brix A, Torre P, Viskum K. Longterm effect of inhaled budesonide in mild and moderate chronic obstructive pulmonary disease: a randomised controlled trial. Lancet 1999; 355: 1819-1823

32 Paggiaro PL, Dahle R, Bakran I, Frith L, Hollingworth K, Efthimiou J. Multicentre randomised placebo-controlled trial of inhaled fluticasone propionate in patients with chronic obstructive pulmonary disease. Lancet 1998; 351: 773-780

33 Burge PS, Calverley PMA, Jones PW, Anderson JA, Maslen TK. Randomised, double blind, placebo controlled study of fluticasone propionate in patients with moderate to severe chronic obstructive pulmonary disease: the ISOLDE trial. BMJ 2000; 320: $1297-1303$

${ }^{34}$ Barnes PJ. Inhaled Corticosteroids are not beneficial in chronic obstructive pulmonary disease. Am J Respir Crit Care Med 2000; 161: $342-344$

${ }^{35}$ Canadian Thoracic Society Workshop Group. Guidelines for the assessment and management of chronic obstructive pulmonary disease. Can Med Assoc J 1992; 147: 420-428

${ }^{36}$ Wettengel R, Böhning W, Cegla U, Criée C, Fichter J, Geisler L, Fabel H, Köhler D, Konietzko N, Lindemann H, Magnussen H, Matthys H, Meister R, Morr H, Nolte D, Petro W, SchultzeWerninghaus G, Sill V, Sybrecht GW, Wiesner B, Worth H. Empfehlungen der Deutschen Atemwegsliga zur Behandlung von Patienten mit chronisch obstruktiver Bronchitis und Lungenemphysem. Med Klinik 1995; 90: 3-7

${ }^{37}$ Fujimoto K, Kubo K, Yamamoto H, Yamaguchi S, Matsuzawa Y. Eosinophilic inflammation in the airway is related to glucocorticoid reversibility in patients with pulmonary emphysema. Chest 1999; 115: 697-702

${ }^{38}$ Schayck van CP, Broek van den PJ, Otter den JJ, Herwaarden van CLA, Molema J, Weel van C. Periodic treatment regimens with inhaled steroids in asthma or chronic obstructive pulmonary disease. Is it possible? JAMA 1995; 274: 161 - 164

${ }^{39}$ Emerman CL, Connors AF, Lukens TW, May ME, Effron D. A randomised controlled trial of methylprednisolone in the emergency treatment of acute exacerbations of chronic obstructive pulmonary disease. Chest 1989; 95: $563-567$

${ }^{40}$ Bullard MJ, Liaw SJ, Tsai YH, Min HP. Early corticosteroid use in acute exacerbations of chronic airflow limitation. Am J Emerg Med 1996; 14: 139-143 
${ }^{41}$ Albert RK, Martin TR, Lewis SW. Controlled trial of methylprednisolone in patients with chronic bronchitis and acute respiratory insufficiency. Ann Intern Med 1980; 92: 753-758

42 Thompson WH, Nielson CP, Carvalho P, Charan NB, Crowley JJ. Controlled trial of oral prednisolone in outpatients with acute COPD exacerbations. Am J Respir Crit Care Med 1996; 154: 407 412

${ }^{43}$ Niewoehner DE, Erbland ML, Deupree RH, Collins D, Gross NJ, Light RW, Anderson P, Morgan NA. Effect of systemic glucocorticoids on exacerbations of chronic obstructive pulmonary disease. N Engl J Med 1999; 340: 1941 - 1947

${ }^{44}$ Davies L, Angus RM, Calverley PMA. Oral corticosteroids in patients admitted to hospital with exacerbations of chronic obstructive pulmonary disease: a prospective randomised controlled trial. Lancet 1999; 354: 456-460

${ }^{45}$ Bristih Thoracic Society. The british guidelines on asthma management. Asthma in adults and schoolchildren. Thorax 1995; 52: S2-S8

\section{Prof. Dr. A. Gillissen}

Med. Universitätsklinik und Poliklinik II

Sigmund-Freud-Str. 25

53105 Bonn

E-mail: adrian.gillissen@mailer.meb.uni-bonn.de 